\title{
Research on Performance Evaluation System Based on Process Model Theory
}

\author{
Zhengqiu Weng ${ }^{1, a^{*}}$, Qiming Tian ${ }^{1, b}$ \\ ${ }^{1}$ WenZhou Vocational \& Technical College, China \\ aderisweng@163.com, btqm78@126.com
}

Keywords: PES (Performance Evaluation System); Process Model; Decision Support

\begin{abstract}
As an important component of the Business Intelligence, Performance Evaluation plays an important role in BI architecture. There are many performance evaluations methods, but people pay less attention to the performance evaluation process that will improve the evaluated objects achievement. According to above reasons, this paper put forward a PES (performance evaluation system) using process model basing on studying performance evaluation theory. Its purpose is to manage the continuous evaluation process by process model and to inspire the evaluated object to archive the goals. Firstly, this paper introduces the performance evaluation theory briefly. Secondly, it precedes detail analysis for process model method and process model core. Finally, this paper shows the result of the PES by applied case and experiment.
\end{abstract}

\section{Introduction}

In order to improve the adaptability to the external environment as well as the speed and efficiency of decision-making, the enterprise internal appears a new form of organization -multi-organizational team which communicates more easily. This form of organization is relatively complete and easier to define the relationship between team performance and overall organizational performance. Commonly, we use performance evaluation to assess each team's performance. [1]

Using certain methods, a particular indicators system and the uniform evaluation criteria, performance evaluation makes objective, fair and accurate comprehensive evaluation in a certain period of management efficiency according certain procedures.

Performance evaluation is an integral part of BI system, which together with data warehouse, ETL, an OLAP analysis to constitute a complete BI system. It makes the data and evaluation indicators together in the data warehouse, and the data analysis and business closely together. $[2,3]$

\section{Problems in the PES}

The problem in the most of the PES is that it focuses only on the performance evaluation methods, such as, performance evaluation accounting index method, Economic Value Added EVA, strategic orientation of the Balanced Scorecard, comprehensive performance evaluation methods, etc. These methods evaluate the process at a point in time for a period of business performance evaluation, but no trace, no attention to the whole process of performance evaluation of multiple periods, also failed to achieve enterprise another final process goals -- the object being evaluated can become outstanding objects in addition to the evaluation of enterprise status. Performance evaluation process management using vertical time axis in my system is an interactive evaluation process[4]. The management of the evaluation process time axis is through the core algorithm of the system - the process model. Using of this model we can manage the continuous evaluation process and stimulate the evaluation object to improve performance. Next we will introduce the core algorithm of this system [5]. 


\section{The key algorithms of process model}

\subsection{Basic Principles of Evaluation Process Model}

The basic idea of this model is based on the "give-and-take" process model, to establish a series of evaluation locus that contains no assessment circumstances, then to describe the characteristics of these evaluations locus.

\subsection{Evaluation process variable modeling}

Promise 1: Evaluation process modeling is defined as follows:

- Collection of objects to be evaluated is $K, K=(1,2, \cdots, n)$

- Evaluation will use different proposal description which will be represented by the set of symbols as follows:

$\mathrm{I}_{k} \subset R^{n}$ ( $\mathrm{N}$ is the number of evaluation index $)$

Represents a proposed description collection of the k-th evaluation object;

- The evaluation object $\mathrm{K}$ will select proposal $\mathrm{T}_{k}\left(\mathrm{~T}_{k} \in \mathrm{I}_{k}\right)$ from $\mathrm{I}_{k}, \mathrm{~T}_{k}$ is he elements of the collection.

Promise 2:

- The proposal description of the evaluation objects in time $t$ is defined as follows:

$\mathbf{I}_{t}=\bigcup_{i=1}^{n} \mathbf{I}_{i, t} \mathbf{t}>0$

- Set performance gap model of all evaluation objects is monotonically decreasing;

- Setting the information obtained from each evaluation is $\mathrm{N}$-dimensional vector. The dimension $i$ is an element of $I_{k}$.

\section{Application Example of Evaluation Process Model}

\subsection{Qualitative interpretation and the actual meaning of the Process Model}

During the evaluation, information will be changed with the evaluation time and the situation changing, so the interaction of the evaluation process is dynamic processes, which will be reflected in the following model, shown in Figure 1.

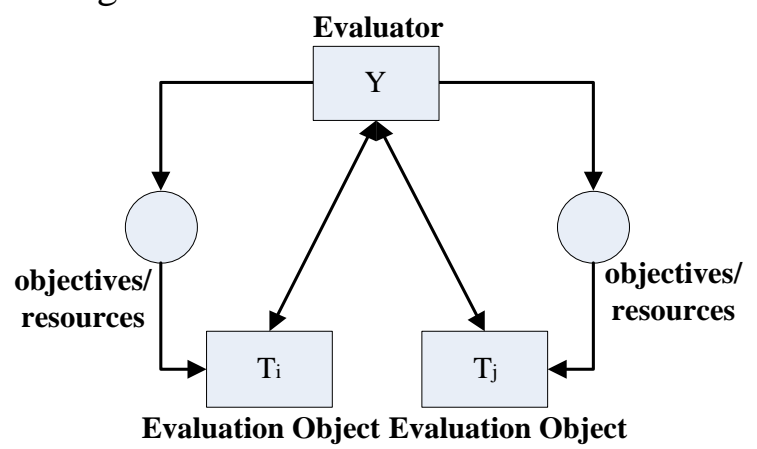

Fig. 1 the Mutual Essentials of Evaluation Process

As shown in Figure 1, the evaluation process between evaluator and the two evaluation objects ( $\mathrm{T} i$ and $\mathrm{T} j$ ) can be described as follows: all recommended information pair for $(\mathrm{T}, \mathrm{Y}) \in$ $\mathrm{I}: \mathrm{I}_{t} * \mathrm{I}_{y}$, among them, $\mathrm{T}_{i} \in \mathrm{I}_{i}, \mathrm{Y} \in \mathrm{I}_{y}$, where the function $\mathrm{F}$ constituted by the by $F_{t}$ and $F_{y}$, in evaluation process, evaluation object will continue to present new information, the space $\mathrm{I}$ is evaluated table space. Thus, a simple individual interaction function can be expressed as:

$$
\begin{aligned}
& F t\left(T_{n}, Y_{n}\right)=k * Y_{n}=T_{n+1} \\
& F_{y}\left(T_{n}, Y_{n}\right)=k * T_{n}=Y_{n+1}(\mathrm{k}<1)
\end{aligned}
$$

The evaluation function $\mathrm{F}$ determines the dynamic process based on the information, $\mathrm{F}$ defines the transformation on the set of I. it points out how to transform from a given state $\left(T_{n}, Y_{n}\right)$ into 
another state $\left(T_{n+1}, Y_{n+1}\right)$, which promotes interactive process in the information space. The bidirectional arrow from $\mathrm{T}$ to $\mathrm{Y}$ indicates the chain of causality.

In this model, we indicate the monotonic of function introducing the polar in mapping geometry. Positive polarity represent incremental, negative polarity indicates descending. So we can use the polar to describe the nature of evaluation function abstractly. There are only two chains between evaluator and the evaluation object. So there exists three kind of combination of chain polarity: positive-positive, positive- negative and negative-negative.

(1) $F_{x}$ and $F_{y}$ have positive polarity

The values of $\mathrm{T}$ and $\mathrm{Y}$ increase or decrease simultaneously, which will occur in two situations: convergence or non-convergence until it reaches a natural limit. This interaction is enhanced (escalatory), which is commonly occurred in the evaluation process. In this evaluation process on the distribution of limited resources, it means a "win - win" situation.

(2) $F_{x}$ and $F_{y}$ have negative polarity

In this case, $\mathrm{T}$ and $\mathrm{Y}$ are also enhanced, but the enhancement of $\mathrm{T}$ and $\mathrm{Y}$ are complementary. This enhancement may be convergent, i.e. the changes of $\mathrm{X}$ and $\mathrm{Y}$ become smaller. it may be not convergent, interactive process continues until a natural limit achieved between $\mathrm{T}$ and $\mathrm{Y}$. It means a "lose - lose" situation, which is rarely exist in the evaluation process.

(3) $F_{x}$ and $F_{y}$, a positive polarity, the other a negative polarity

In this case, the interactive evaluation process will always oscillating, or decay to stable values of $\mathrm{T}$ and $\mathrm{Y}$.

In summary, the evaluation function can be divided into enhanced (positive polarity) and nonenhanced (negative polarity). So we introduce the limited objectives/resources in the performance evaluation process, making the process of convergence, which will change the "lose - lose" situation to "win - win" situation.

\subsection{Example Analysis of the Process Model}

Here we use computer to record, display and interpret evaluation rail line, and explain how it assists analysis and description of the evaluation object. In this example, there are only two issuesfinancial benefits and asset operations, and then introduces the issue of solvency.

As shown in Figure 2 to Figure 4, the rise rectangular frame shows gradually increasing about evaluation locus. Figure 2 and Figure 3 show the evaluation of the evaluation object at different times. Figure 4 shows two-dimensional space from the point of view of the evaluation object. Similarly, we can also introduce other new issues.

The reduction of collection space can be detected by the compression factor. On each dimension there are three compression factors:

The reduction of collection space can be detected by the compression factor. On each dimension there are three compression factors:

When there is unilateral improvement in financial benefit, the compression factor of this stage will be less than 1 ;

When there is no unilateral improvement in financial benefits, compression factor will equal 1;

If withdraw the previous concessions, the compression factor will be more than 1 .

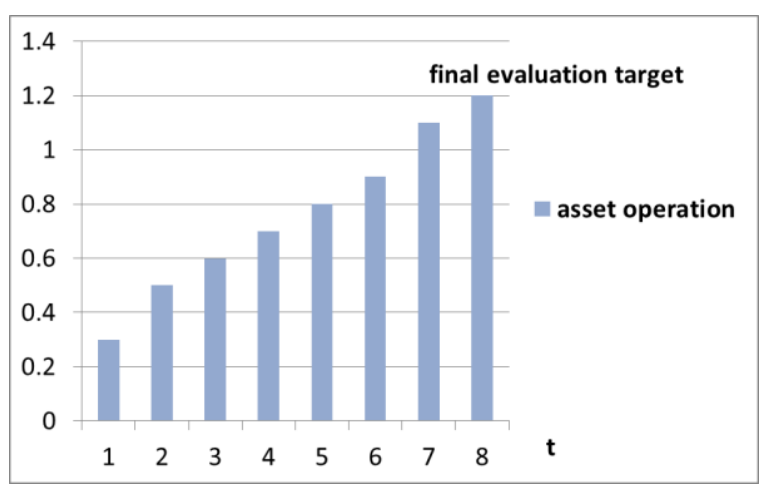

Fig. 2 Evaluation Locus of Asset Operation

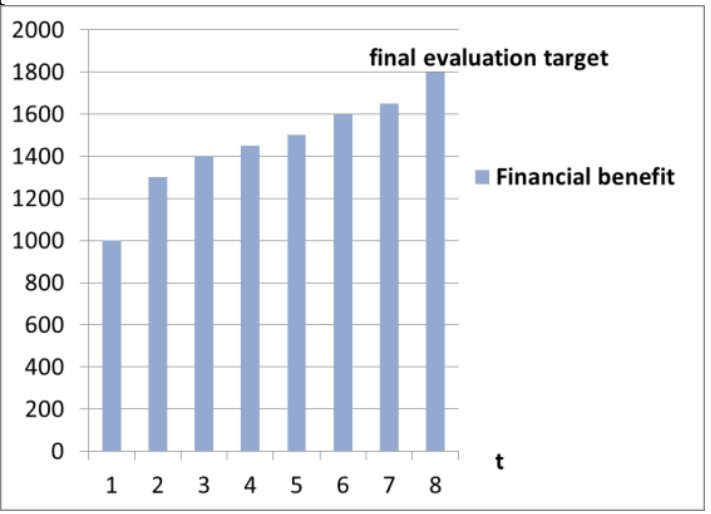

Fig. 3 Evaluation Locus of Financial benefit 


\subsection{Test results}

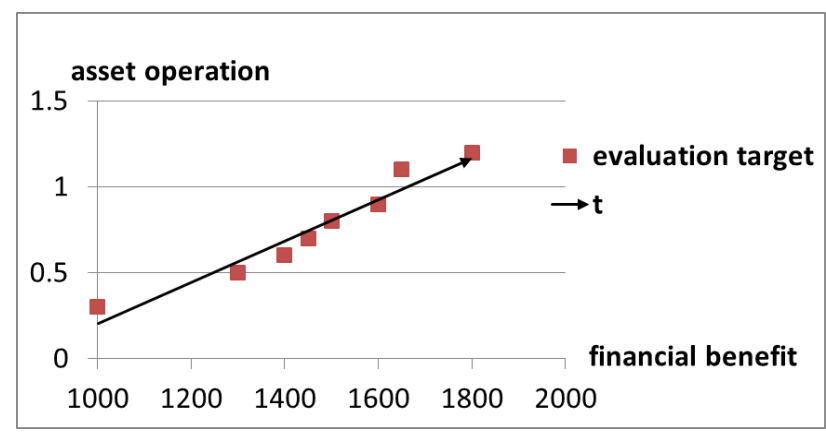

Fig. 4 Evaluation of two-dimensional space

In this evaluation model, the evaluation objects take concessions in the process. When all evaluation objects take no concessions and are unable to reach a "win - win" situation, the evaluator will take concessions. Evaluation results are listed in Table 1, which shows the excellent rate of enterprise evaluation after the application of this system.

Evaluation object number: 63

The evaluation period: 1 month

The evaluation observe time: 12 months

Table 1 the excellent rate of enterprise evaluation

\begin{tabular}{|l|l|l|l|l|l|l|l|l|l|l|l|l|}
\hline month & 1 & 2 & 3 & 4 & 5 & 6 & 7 & 8 & 9 & 10 & 11 & 12 \\
\hline excellent rate & $42 \%$ & $45 \%$ & $46 \%$ & $43 \%$ & $56 \%$ & $55 \%$ & $63 \%$ & $49 \%$ & $57 \%$ & $65 \%$ & $66 \%$ & $69 \%$ \\
\hline
\end{tabular}

\section{Conclusions}

Through this study, the performance of evaluation system is improving based on the management of evaluation process. Application of the process model, the PES can not only use typical methods on evaluation objects, but can also be formed by the interaction of the object model and evaluation process. After a time course evaluation, the ability and level of evaluation object is improved. On the other hand, due to the complexity of the actual performance evaluation and limited time, the system needs further improvement and research. For example, there is obviously irregular point in test data. The causes and how to control irregular points, as well as how to accelerate the speed of convergence remains to be evaluated by further discussion.

\section{Acknowledgment}

This work is supported by Zhejiang province Higher Vocational College Professional Leaders' Leading Project (No. lj2013043), all support is gratefully acknowledged.

\section{References}

[1] Gao Ying-hui. Study on evaluation system of government debt sustainability of resource-exhausting regions, Management Science \& Engineering (ICMSE). (2014)

[2] Wang Yinming, Zhao Xihui, etc., Study on performance evaluation system of renewable energy fields, Service Operations and Logistics, and Informatics (SOLI). (2013)

[3] Jingmin Wang, etc., Research of the design of the ERP application evaluation system based on the module method, Mechatronic Science, Electric Engineering and Computer (MEC). (2011)

[4] Feng Qing. Key Technology Research on Generalized Product Knowledge based Decision Support System, Northwestern Polytechnical University. (2014)

[5] ZHU Shunnan, Evaluation System and Econometric Model on Dynamic Performance of Technology Transfer, Scientific management research. 31 (2013). 